\title{
Protective effects of dehydrocostuslactone on rat hippocampal slice injury induced by oxygen-glucose deprivation/reoxygenation
}

\author{
QIPENG ZHAO ${ }^{1,2^{*}}$, AILING CHEN $^{1,2^{*}}$, XIAOBO WANG $^{1,3}$, ZHUANZHUAN ZHANG $^{1,2}$, \\ YUNSHENG ZHAO $^{1,2}$, YU HUANG ${ }^{2}$, SHUANGLAI REN $^{4}$ and YAFEI ZHU ${ }^{1}$ \\ ${ }^{1}$ Key Laboratory of Hui Ethnic Medicine Modernization, Ministry of Education, Ningxia Medical University; \\ ${ }^{2}$ Department of Pharmacology, Ningxia Medical University, Yinchuan, Ningxia Hui Autonomous Region 750004; \\ ${ }^{3}$ Department of Pharmacology, Chengdu University of TCM, Chengdu, Sichuan 610075; ${ }^{4}$ Ningxia Key Lab of Craniocerebral \\ Diseases of Ningxia Hui Autonomous Region, Yinchuan, Ningxia Hui Autonomous Region 750004, P.R. China
}

Received October 22, 2017; Accepted May 15, 2018

DOI: $10.3892 / \mathrm{ijmm} .2018 .3691$

\begin{abstract}
The present study aimed to investigate the protective effects of dehydrocostuslactone (DHL) against rat hippocampal slice injury caused by oxygen-glucose deprivation/reoxygenation (OGD/R). Rat hippocampal slice injury was induced by $\mathrm{OGD} / \mathrm{R}$ in vitro, and the degree of injury was evaluated through a lactate dehydrogenase (LDH) assay and 2,3,5-triphenyltetrazolium chloride (TTC) staining. The protein expression levels of B-cell lymphoma-2 (Bcl-2), Bcl-2-associated X protein (Bax), cytochrome $c$ (cyt-c), apoptotic protease activating factor 1 (apaf-1), caspase-9, caspase-7, caspase-3, sequestosome 1 (SQSTM1) and microtubule-associated protein 1 light chain 3 (LC3) were analyzed through western blot analysis. The results showed that 1,5 and $10 \mu \mathrm{M}$ DHL decreased the levels of LDH $(\mathrm{P}<0.05)$ and increased the $\mathrm{A}_{490}$ value of TTC $(\mathrm{P}<0.05)$. Furthermore, the expression of $\mathrm{Bcl}-2$ was enhanced, and the protein expression levels of Bax, cyt-c, apaf-1, caspase-9, caspase-7, caspase-3, SQSTM1 and LC3 were significantly inhibited $(\mathrm{P}<0.05)$, compared with those in the OGD/R group. These results suggested that DHL elicited protective effects against hippocampal OGD/R injury, and its underlying mechanism may be associated with inhibiting apoptosis.
\end{abstract}

Correspondence to: Miss Yafei Zhu, Key Laboratory of Hui Ethnic Medicine Modernization, Ministry of Education, Ningxia Medical University, 1160 Shengli Street, Xingqing, Yinchuan, Ningxia Hui Autonomous Region 750004, P.R. China

E-mail: zhyafei1214@126.com

Mr. Shuanglai Ren, Ningxia Key Lab of Craniocerebral Diseases of Ningxia Hui Autonomous Region, Ningxia Medical University, 1160 Shengli Street, Xingqing, Yinchuan, Ningxia Hui Autonomous Region 750004, P.R. China

E-mail: renshuanglai@sina.com

*Contributed equally

Key words: dehydrocostuslactone, hippocampal brain slices, oxygen-glucose deprivation/reoxygenation, apoptosis, autophagy

\section{Introduction}

Ischemic stroke is a common cause of mortality worldwide $(1,2)$. The main pathogenetic mechanisms of cerebral ischemia include intracellular calcium overload $(3,4)$, excitatory amino acid toxicity, increase in oxygen free radicals (4), inflammatory responses $(4,5)$, autophagy and apoptosis $(6)$. A complex interplay also exists among these factors, for example, autophagy and apoptosis. Although a suitable level of autophagy may provide neuroprotection, the excessive activation of autophagy can induce cell death. Apoptosis is essential in the pathogenesis and prognosis of different cerebrovascular diseases, including chronic and acute ischemia. As a result of hypoxia and lack of glucose, when cells suffer from ischemic stimulation due to an increasing number of reactive oxygen species (ROS) in the cytoplasm (7), including $\cdot \mathrm{O}_{2}-\mathrm{H}_{2} \mathrm{O}_{2}$ and $\cdot \mathrm{OH}$, in addition to anti-apoptotic protein $\mathrm{B}$-cell lymphoma-2 (Bcl-2) and pro-apoptotic protein Bcl-2-associated X protein (Bax) $(8,9)$, the mitochondrial membrane permeability transition pore is opened. This leads to increased mitochondrial membrane permeability and decreased mitochondrial membrane potential. Subsequently, cytochrome $c$ (cyt-c) is translocated from the mitochondrial matrix to the cytoplasm, forming an apoptotic complex by combining apoptotic protease activating factor 1 (apaf-1) and caspase-9. Finally, the caspase-dependent apoptotic pathway is activated. Furthermore, other transcription factors, including, $\mathrm{p} 53$, tumor necrosis factor- $\alpha$ and nuclear factor- $\mathrm{\kappa} B$, may be involved in the apoptotic process $(10,11)$. Therefore, inhibiting autophagy and apoptosis is a possible target for stroke treatment.

The hippocampus is a region of the brain sensitive to changes in oxygen-glucose levels (12). Rat hippocampal slices are used in in vitro experiments, as a mutual connection between neurons and glial cells is maintained $(13,14)$. Hippocampal slices have been used to investigate the effects of anoxia, excitotoxicity and depolarization (15-17). The excitability of CA1 neurons increases when cerebral ischemia occurs. If oxygen-glucose supply is not restored, or therapeutic windows exceed optimal levels, neurons become excited until these cells die (18). 
Dehydrocostuslactone (DHL) is a sesquiterpene lactone derived from the medicinal plant Aucklandia lappa Dence, belonging to the family Asteraceae (19) and is a major active ingredient of Aucklandiae Radix. The metabolite of DHL can penetrate the blood-brain barrier and exhibit various pharmacological activities, including anti-inflammatory (20), anti-ulcer (19) and antimicrobial properties (21), anti-apoptotic and pro-apoptotic effects on different human cancer cell lines $(22,23)$, and oxidative and endoplasmic reticulum stress responses (24).

In the present study, rat hippocampal slices were subjected to oxygen-glucose deprivation and reoxygenation (OGD/R) and used to investigate whether DHL reduces neuronal apoptosis and autophagy in vitro.

\section{Materials and methods}

Animals. Male Sprague-Dawley rats (weighing 250-290 g, aged 8-10 weeks old) were purchased from the Experimental Animal Center of Ningxia Medical University [Yinchuan, China; animal license no. SCXK (Ning) 2011-0001]. All rats were maintained in a well-ventilated environment with a $12 \mathrm{~h} / 12 \mathrm{~h}$ light/dark cycle at constant room temperature $\left(25 \pm 2^{\circ} \mathrm{C}\right)$ and humidity $(50-70 \%)$. The rats were provided with free access to normal rodent food and water.

Reagents. DHL (cat. no. 34080) was purchased from Chengdu Pusi Biological Technology Co., Ltd. (Chengdu, China). A stock solution was prepared using dimethyl sulfoxide (DMSO) solution and diluted with normal artificial cerebrospinal fluid (aCSF) containing $124 \mathrm{mM} \mathrm{NaCl}, 3.00 \mathrm{mM} \mathrm{KCl}, 2.50 \mathrm{mM}$

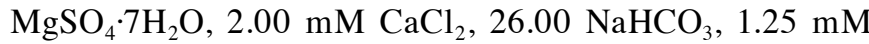
$\mathrm{NaH}_{2} \mathrm{PO}_{4}$ and $10.00 \mathrm{mM}$ glucose, and glucose-free aCSF (equal mole sucrose instead of glucose) to obtain the required concentration. Low, middle and high DHL concentrations were set at 1,5 and $10 \mu \mathrm{M}$, respectively. Nimodipine injection (cat. no. H20140301) was provided by Bayer Schering Pharma AG (Shanghai, China). The nimodipine concentration was set at $10 \mu \mathrm{M}$. 2,3,5-triphenyltetrazolium chloride (TTC; cat. no. T8877; Sigma-Aldrich; EMD Millipore, Billerica, MA, USA) was dissolved in aCSF and stored in the dark. The following substances and kits were utilized in the present study: Lactate dehydrogenase (LDH) assay kit (cat no. A020-2; Nanjing Jiancheng Bioengineering Institute, Nanjing, China); total protein extraction and BCA protein quantification kits (Nanjing KeyGen Biotech, Co., Ltd., Nanjing, China); primary antibodies against Bcl-2 (cat. no. 2876), Bax (cat. no. 2772), cyt-c (cat. no. 4272), apaf-1 (cat. no. 8723) and $\beta$-actin (cat. no. 4970) were obtained from Cell Signaling Technology, Inc., (Danvers, MA, USA); primary antibodies against caspase-9 (cat. no. ab2013), caspase-7 (cat. no. ab25900) and caspase-3 (cat. no. ab44976) were procured from Abcam (Cambridge, UK); primary antibodies against sequestosome 1 (SQSTM1; cat. no. bs-2951R) and microtubule-associated protein 1 light chain 3 (LC3; cat. no. bs-8878R) were procured from BIOSS (Beijing, China); horseradish peroxidase-conjugated goat anti-rabbit IgG secondary antibody (cat. no. ZB-2301) was obtained from ZSGB-BIO (Beijing, China); Z-VAD-FMK, a novel broad-spectrum caspase protease inhibitor (cat. no. C1202) was obtained from Beyotime Institute of Biotechnology (Nantong, China).
Rat hippocampal slice preparation. The rats were anesthetized with $7 \%$ chloral hydrate $(350 \mathrm{mg} / \mathrm{kg}$, i.p.) and sacrificed by decapitation. Their brains were rapidly excised and placed in an ice-cold bath filled with a $95 \% \mathrm{O}_{2} / 5 \% \mathrm{CO}_{2}$ gas mixture of aCSF for $1 \mathrm{~min}$. The bilateral hippocampus was also rapidly excised. The hippocampal slices were isolated, coronally trimmed, glued to the stage of a vibration slicing machine (LEICA VT 1000S; Leica Microsystems, Inc., Buffalo Grove, IL, USA), and coronally cut to a thickness of $400-\mu \mathrm{m}$ at $0^{\circ} \mathrm{C}$. The hippocampal slices were divided into eight groups: Control, OGD, OGD+nimodipine $(10 \mu \mathrm{M}$, dissolved in aCSF and glucose-free aCSF), OGD+DHL (1, 5 and $10 \mu \mathrm{M}$ dissolved in DMSO, aCSF and glucose-free aCSF, respectively), OGD+Z-VAD-FMK $(20 \mu \mathrm{M})$, and OGD+Z-VAD-FMK $(20 \mu \mathrm{M})+\mathrm{DHL}(10 \mu \mathrm{M})$. Each group contained 40 slices. All hippocampal slices were immediately pipetted lightly into an incubator and then recovered for $1 \mathrm{~h}$ in oxygenated aCSF at $37^{\circ} \mathrm{C}$.

$O G D / R$ injury model. Following the recovery period, the slices were lightly washed with aCSF and transferred into a 24-well culture plate with four slices per well. The OGD of the experimental groups was induced by glucose-free aCSF. This dissection buffer was bubbled with a $95 \% \mathrm{~N}_{2} / 5 \% \mathrm{CO}_{2}$ gas mixture in a 24-well culture plate for $30 \mathrm{~min}$. The control group was incubated in aCSF equilibrated with $95 \% \mathrm{O}_{2} / 5 \% \mathrm{CO}_{2}$ for $30 \mathrm{~min}$ (OGD period). Subsequently, the slices were transferred into a new 24-well culture plate containing oxygenated normal aCSF and placed in a $95 \% \mathrm{O}_{2} / 5 \% \mathrm{CO}_{2}$ gas mixture incubator. Finally, the slices were incubated for $1 \mathrm{~h}$ at $37^{\circ} \mathrm{C}$. Five treatment groups received nimodipine $(10 \mu \mathrm{M})$, Z-VAD-FMK $(20 \mu \mathrm{M})$, DHL $(1,5$ and $10 \mu \mathrm{M})$, and Z-VAD-FMK $(20 \mu \mathrm{M})+\mathrm{DHL}$ $(10 \mu \mathrm{M})$ within the OGD/R period. The drugs were dissolved in $\mathrm{aCSF}$ and glucose-free aCSF.

LDH activity measurement. $\mathrm{LDH}$ is a cytosolic enzyme that can be released into extracellular fluid when cell membranes are disrupted. Therefore, this enzyme is used to evaluate the degree of injury. In the present study, the viability of the rat hippocampal slices was determined following the completion of reoxygenation by measuring the concentration of $\mathrm{LDH}$ released into the culture supernatants through spectrophotometry at $450 \mathrm{~nm}$ in an ELISA reader in accordance with the manufacturer's protocol.

TTC staining. Following reoxygenation completion, the rat hippocampal slices were transferred into small vials $(50 \mathrm{ml})$ containing $2 \%(\mathrm{w} / \mathrm{v})$ TTC solution in aCSF aerated vigorously for $10 \mathrm{~min}$ at $37^{\circ} \mathrm{C}$. The stained slices were subsequently removed and rinsed twice with normal saline. The slices were dried and weighed. An extraction buffer (DMSO and 100\% ethanol prepared 1:1) was added (20 $\mu \mathrm{l} / \mathrm{mg})$ and stored in the dark for $24 \mathrm{~h}$ at room temperature. The quantity of the extracted formazan was then measured through spectrophotometry at $490 \mathrm{~nm}$ using an ELISA reader.

Western blot analysis. Western blot analysis was performed to detect the relative levels of Bcl-2, Bax, cyt-c, apaf-1, caspase-9, caspase-7 and caspase-3. The slices were treated and grouped as indicated in the previous section. In each group, four slices were collected as one sample following administration of the 
respective treatments. All samples were rapidly placed in an ice bath, and total protein extraction was initiated using the total protein extraction kit. The protein extraction fluid, containing lysis buffer $(1 \mathrm{ml})$, PMSF $(10 \mu \mathrm{l})$, protein inhibitor $(1 \mu \mathrm{l})$ and phosphatase inhibitor $(10 \mu \mathrm{l})$, was added in a volume of $0.5 \mathrm{ml}$ to $100 \mathrm{mg}$ of tissue slice in a Pro homogenizer and homogenized 30 times. The homogenate was transferred to a centrifuge tube and centrifuged at $12,000 \mathrm{xg}$ for $10 \mathrm{~min}$ at $4^{\circ} \mathrm{C}$ to remove cellular debris. The BCA protein assay reagent kit was used to determine the protein concentration. The lysates from the slices were boiled for $5 \mathrm{~min}$. Equivalent quantities $(80 \mu \mathrm{g})$ of protein samples were subjected to $10 \%$ SDS-PAGE and electrically transferred onto PVDF membranes using an electrophoretic transfer system (40D; Beijing Liuyi Biotechnology Co., Ltd., Beijing, China). These membranes were subdivided, and each target protein and loading control protein were analyzed from a single transfer. Nonspecific binding sites were blocked with PBST containing 5\% nonfat dry powdered milk for $1 \mathrm{~h}$ at room temperature. The membranes were then incubated with primary antibodies against Bcl-2 (1:1,000), Bax $(1: 3,00)$, cyt-c $(1: 1,000)$ apaf-1 $(1: 1,000)$, caspase-9 $(1: 1,000)$, caspase-7 $(1: 500)$, caspase-3 $(1: 500)$, and $\beta$-actin $(1: 1,000)$ at $4^{\circ} \mathrm{C}$ overnight. Immunostained $\beta$-actin served as the respective control. The membranes were washed three times with PBST containing $0.1 \%$ Tween and re-incubated with horseradish peroxidase-conjugated secondary antibodies $(1: 2,000)$ for $2 \mathrm{~h}$ at room temperature. The bands were then visualized using a Super Signal West Pico Chemiluminescence kit and finally exposed to radiographic films. The resulting bands were analyzed with Quantity One software (version 4.62; Bio-Rad Laboratories, Inc., Hercules, CA, USA). The image intensities of Bcl-2, Bax, caspase-9, caspase-7, caspase-3, SQSTM1 and LC3 were quantified and normalized with $\beta$-actin protein band density.

Statistical analysis. Statistical analysis was performed using SPSS 17.0 (SPSS, Inc., Chicago, IL, USA). The results are presented as the mean \pm standard deviation. Differences among more than two groups were assessed using one-way analysis of variance followed by the least significant difference post hoc test. Differences between two groups were analyzed using an unpaired $t$-test. $\mathrm{P}<0.05$ was considered to indicate a statistically significant difference.

\section{Results}

Effects of DHL on LDH release in OGD/R-injured rat hippocampal slices. The degree of injury on the hippocampal slices exposed to $\mathrm{OGD} / \mathrm{R}$ was determined through the $\mathrm{LDH}$ assay. The OGD/R group showed a significant increase in LDH catalytic activity compared with that of the control group. The LDH concentrations of the DHL-treated groups $(1,5$ and $10 \mu \mathrm{M})$ were lower than that of the OGD/R group $(\mathrm{P}<0.05)$, and this finding was concentration-dependent. No significant differences in the levels of LDH were observed between the nimodipine and DHL groups (1, 5 and $10 \mu \mathrm{M} ; \mathrm{P}>0.05$; Fig. 1).

Effects of DHL on TTC staining of OGD/R-injured hippocampal slices. The TTC staining results were quantified through spectrophotometry at $490 \mathrm{~nm}$ using an ELISA reader.

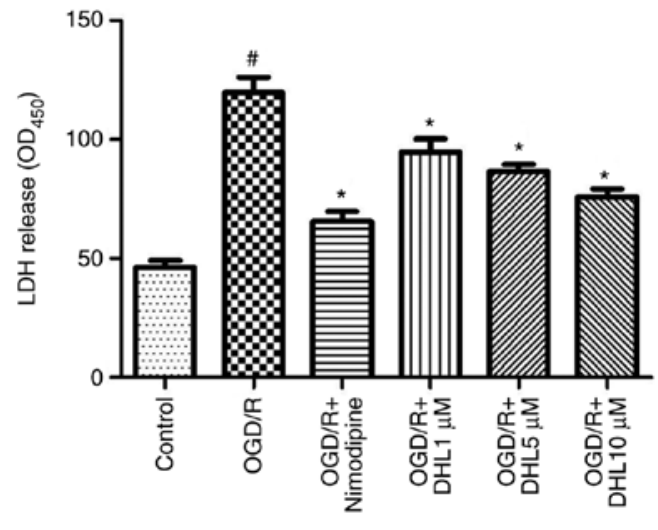

Figure 1. Effect of DHL on LDH release in the culture medium of hippocampal slices subjected to $\mathrm{OGD} / \mathrm{R}$. Values are presented as the mean \pm standard deviation $(\mathrm{n}=10)$. ${ }^{\text {}} \mathrm{P}<0.05$, compared with the control group; ${ }^{*} \mathrm{P}<0.05$, compared with the OGD/R group. DHL, dehydrocostuslactone; $\mathrm{LDH}$, lactate dehydrogenase; OGD/R, oxygen-glucose deprivation/reoxygenation.

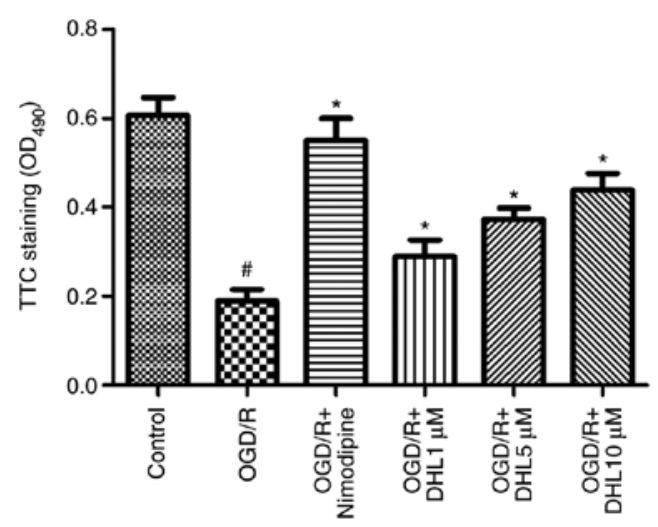

Figure 2. Effect of DHL on TTC staining of OGD/R-injured hippocampal slices. The results showed that DHL increased the $\mathrm{OD}_{490}$ values of rat hippocampal slices subjected to $\mathrm{OGD} / \mathrm{R}$. Values are presented as the mean \pm standard deviation $(\mathrm{n}=10) .{ }^{*} \mathrm{P}<0.05$, compared with the control group; ${ }^{*} \mathrm{P}<0.05$, compared with the OGD/R group. DHL, dehydrocostuslactone; OGD/R, oxygen-glucose deprivation/reoxygenation; TTC, 2,3,5-triphenyltetrazolium chloride.

The $\mathrm{OD}_{490}$ of TTC staining was obtained to evaluate the degree of hippocampal slice injury. The results demonstrated that the $\mathrm{OD}_{490}$ of the $\mathrm{OGD} / \mathrm{R}$ group was significantly lower than that of the control group, the DHL-treated groups, and the nimodipine-treated group. The $\mathrm{OD}_{490}$ of the DHL $(1,5$ and $10 \mu \mathrm{M})$ and nimodipine groups were significantly higher than that of the OGD/R group $(\mathrm{P}<0.05)$, with no significant difference in $\mathrm{OD}_{490}$ was observed between the nimodipine- and DHL-treated groups (1, 5 and $10 \mu \mathrm{M} ; \mathrm{P}>0.05$; Fig. 2).

Effects of DHL on the expression of Bcl-2, Bax, cyt-c, apaf-1, caspase-9, caspase- 7 and caspase- 3 . To investigate the anti-apoptotic effects of DHL on rat hippocampal slice injury induced by $\mathrm{OGD} / \mathrm{R}$, the present study determined the expression levels of cyt-c (Fig. 3), apaf-1 (Fig. 4), Bcl-2, Bax (Fig. 5), caspase-9 (Fig. 6), caspase-7 (Fig. 7) and caspase-3 (Fig. 8), and used these as markers of apoptosis in the mitochondria. The results of western blot analysis revealed that the expression level of Bcl-2 in the OGD/R group was significantly lower than that of the control group, whereas the expression level of 

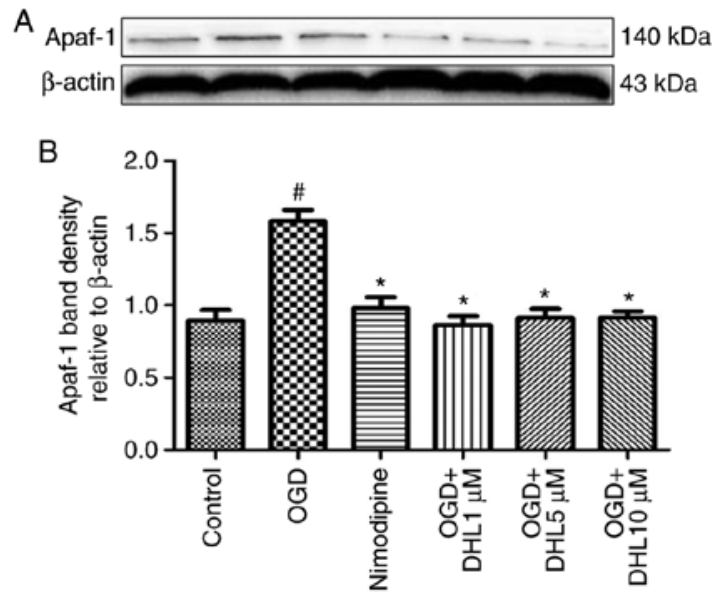

Figure 3. Effects of DHL on the expression of apaf-1. (A) Western blot analysis of apaf-1, with $\beta$-actin shown as a loading control; (B) Quantitative analysis of the expression of apaf-1. Values are presented as the mean \pm standard deviation $(\mathrm{n}=10)$. ${ }^{\#} \mathrm{P}<0.05$, compared with the control group; ${ }^{*} \mathrm{P}<0.05$, compared with the OGD/R group. apaf-1, apoptotic protease activating factor 1; DHL, dehydrocostuslactone; OGD/R, oxygen-glucose deprivation/reoxygenation.

Bax in the OGD/R group was markedly higher than that of the control group. Treatment with DHL $(1,5$ and $10 \mu \mathrm{M})$ enhanced the expression of Bcl-2 and significantly inhibited the expression of Bax. The expression levels of cyt-c, apaf-1, caspase-9, caspase- 7 and caspase- 3 were also significantly increased in the OGD/R group. Treatment with DHL $(1,5$ and $10 \mu \mathrm{M})$ reduced the expression levels of apaf-1, cyt-c, caspase-9, caspase-7 and caspase-3, however, no significant difference was found in the expression levels of Bcl-2, Bax, cyt-c, apaf-1, caspase- 9 , caspase-7 and caspase- 3 between the nimodipineand DHL-treated groups $(1,5$ and $10 \mu \mathrm{M} ; \mathrm{P}>0.05)$.

Effects of Z-VAD-FMK and DHL on the expression of cyt-c, apaf-1, caspase-9, caspase-7 and caspase-3. To compare the anti-apoptotic effects of Z-VAD-FMK and DHL on rat hippocampal slice injury induced by $\mathrm{OGD} / \mathrm{R}$, the present study determined the expression levels of cyt-c, apaf-1, caspase-7 (Fig. 9), caspase-9 (Fig. 10) and caspase-3 (Fig. 11). The results suggested that Z-VAD-FMK downregulated the expression levels of caspase-9, caspase-7 and caspase-3 (1,5 and $10 \mu \mathrm{M} ; \mathrm{P}<0.05)$.

Effects of DHL on the expression of SQSTM1 and LC3-II. To investigate the anti-autophagy effects of DHL on rathippocampal slice injury induced by OGD/R, the present study determined the expression levels of LC3-II (Fig. 12) and SQSTM1 (Fig. 13). The expression levels of LC3-II and SQSTM1 were markedly increased in the OGD/R group compared with that of the control group. Treatment with DHL $(1,5$ and $10 \mu \mathrm{M})$ inhibited the expression of LC3-II and SQSTM1 $(\mathrm{P}<0.05)$.

\section{Discussion}

In the present study, rat hippocampal slices were used to establish an OGD/R model, which is an in vitro model of ischemia $(25,26)$, and ischemic stroke was simulated to investigate the protective effects and mechanisms of DHL.
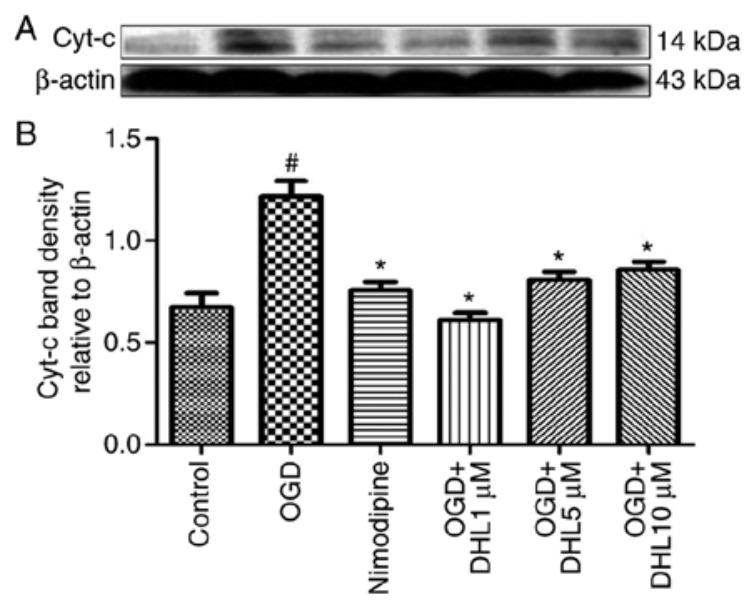

Figure 4. Effects of DHL on the expression of cyt-c. (A) Western blot analysis of cyt-c, with $\beta$-actin shown as a loading control; (B) Quantitative analysis of expression of cyt-c. Values are presented as the mean \pm standard deviation $(\mathrm{n}=10) .{ }^{\#} \mathrm{P}<0.05$, compared with the control group; ${ }^{*} \mathrm{P}<0.05$, compared with the OGD/R group. cyt-c, cytochrome $c$; DHL, dehydrocostuslactone; $\mathrm{OGD} / \mathrm{R}$, oxygen-glucose deprivation/reoxygenation.

Changes in the quality and quantity of LDH directly affect the energy metabolism of the body. LDH can be released into extracellular spaces through a damaged cell membrane if external stimuli, including hypoxia-ischemia, are present (27). Therefore, an LDH assay can be used to quantify the effects of OGD (28). Cechetti et al (29) performed an LDH assay and examined the effects of treadmill exercise on cell damage in rat hippocampal slices subjected to oxygen and glucose deprivation. The LDH assay can also be utilized to evaluate membrane integrity loss and pathological necrosis (30). In addition, TTC staining has been used to examine brain injuries quantitatively (31). In this method, TTC is oxidized only by metabolically active mitochondrial dehydrogenases and converted into red formazan. The quantity of formazan is directly proportional to cell activity (32). For example, TTC staining has been used to measure the area of cerebral infarcts in brain slices, and TTC assays have also been performed to assess cell and tissue viability $(33,34)$. In the present study, the level of released LDH was significantly increased, and the $\mathrm{OD}_{490}$ of TTC staining was significantly reduced when the cells were exposed to OGD/R; this change demonstrated that the hippocampal slices were more vulnerable to OGD/R injury (26). DHL treatment markedly decreased the release of $\mathrm{LDH}$ and increased the $\mathrm{OD}_{490}$ of TTC staining.

To enhance our understanding of the mechanisms underlying the action of $\mathrm{OGD} / \mathrm{R}$, the present study elucidated the levels of apoptosis-related proteins as factors that may be involved in DHL-induced neuroprotection against rat hippocampal slice $\mathrm{OGD} / \mathrm{R}$ damage, as $\mathrm{Bcl}-2$ inhibits programmed cell death without affecting cellular proliferation (35). Bcl-2 was the first anti-apoptotic protein identified with a protective activity against ischemic injury (36). Furthermore, Bax, a pro-apoptotic protein found in the cytosol of healthy cells, promotes apoptosis when cells are stimulated by ischemia $(37,38)$. In addition, Bax can combine with Bcl-2 to form polymers, increase the permeability of the mitochondrial membrane, activate the caspase-apoptosis pathway, and trigger cell apoptosis. The expression levels of Bcl-2 are 


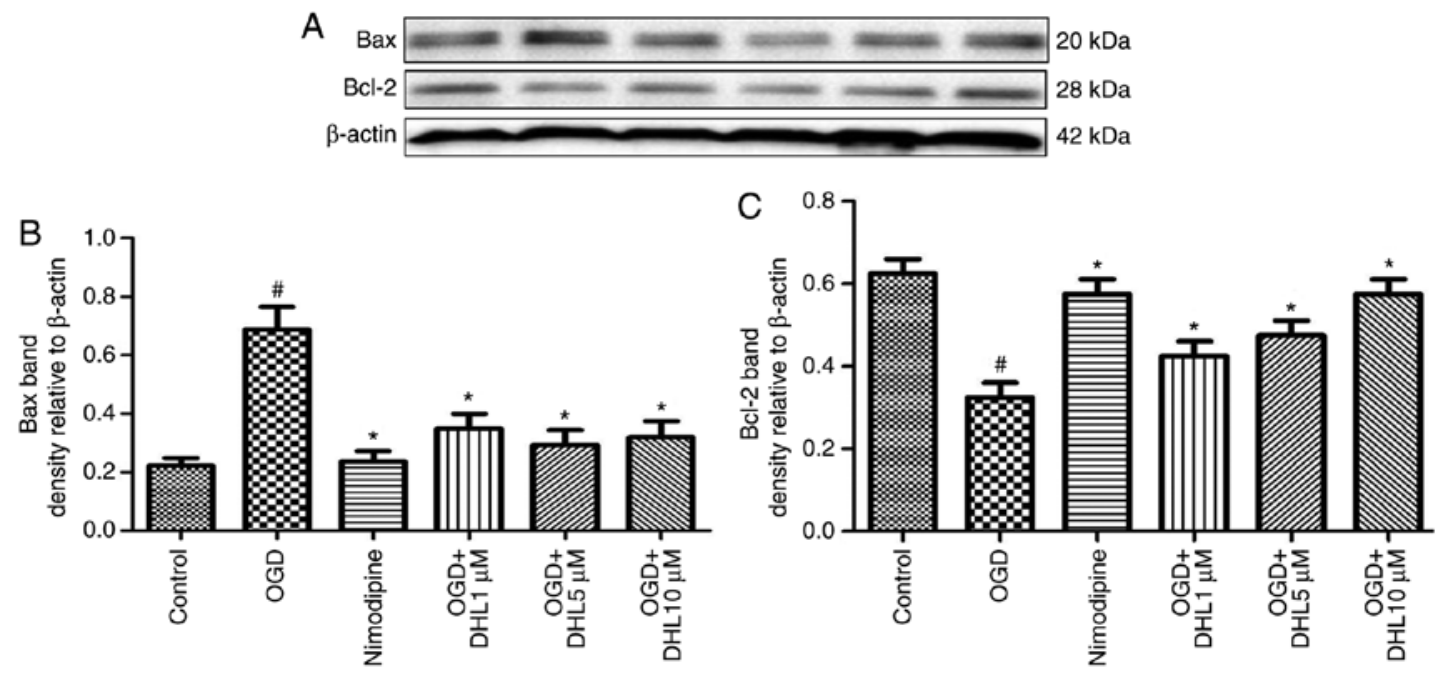

Figure 5. Effects of DHL on the expression of Bax and Bcl-2. (A) Western blot analysis of Bax and Bcl-2, with $\beta$-actin shown as a loading control. (B) Quantitative analysis of the expression of Bax. (C) Quantitative analysis of the expression of Bcl-2. Values are presented as the mean \pm standard deviation ( $\mathrm{n}=10$ ). ${ }^{\#} \mathrm{P}<0.05$, compared with the control group; "P<0.05, compared with the OGD/R group. Bcl-2, B-cell lymphoma-2; Bax, Bcl-2-associated X protein; DHL, dehydrocostuslactone; OGD/R, oxygen-glucose deprivation/reoxygenation.
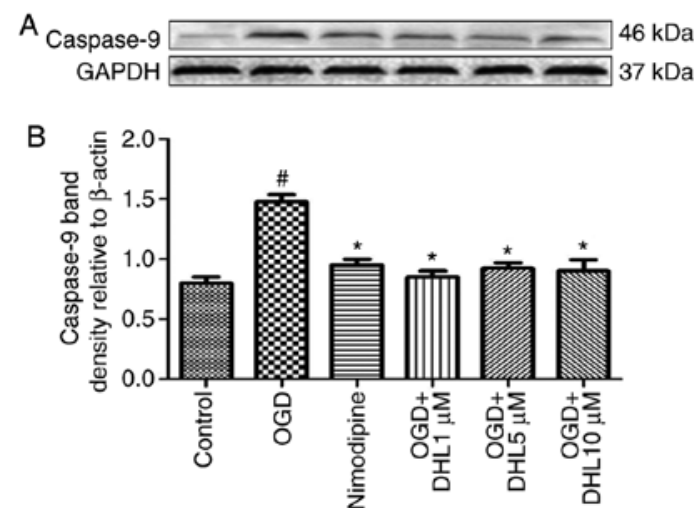

Figure 6. Effects of DHL on the expression of caspase-9. (A) Western blot analysis of caspase-9, with $\beta$-actin shown as a loading control.(B) Quantitative analysis of the expression of caspase-9. Values are presented as the mean \pm standard deviation $(\mathrm{n}=10) .{ }^{*} \mathrm{P}<0.05$, compared with the control group; ${ }^{*} \mathrm{P}<0.05$, compared with the OGD/R group. DHL, dehydrocostuslactone; $\mathrm{OGD} / \mathrm{R}$, oxygen-glucose deprivation/reoxygenation.
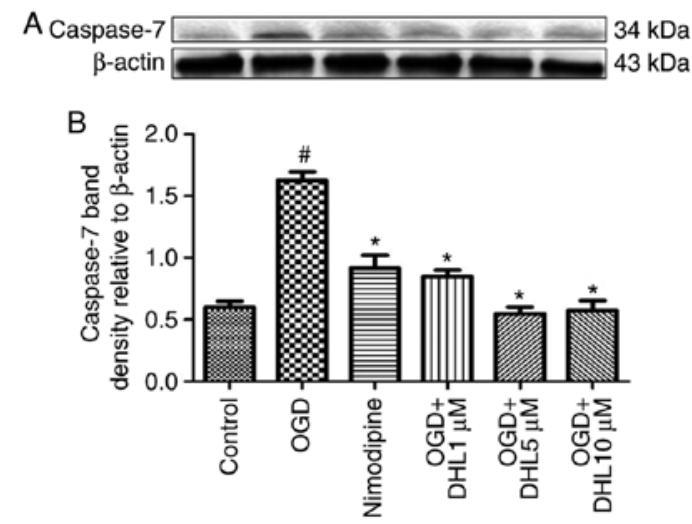

Figure 7. Effects of DHL on the expression of caspase-7. (A) Western blot analysis of caspase-7, with $\beta$-actin shown as a loading control.(B) Quantitative analysis of the expression of caspase-7. Values are presented as the mean \pm standard deviation $(\mathrm{n}=10) .{ }^{\prime \prime} \mathrm{P}<0.05$, compared with the control group; ${ }^{*} \mathrm{P}<0.05$, compared with the OGD/R group. DHL, dehydrocostuslactone; $\mathrm{OGD} / \mathrm{R}$, oxygen-glucose deprivation/reoxygenation.
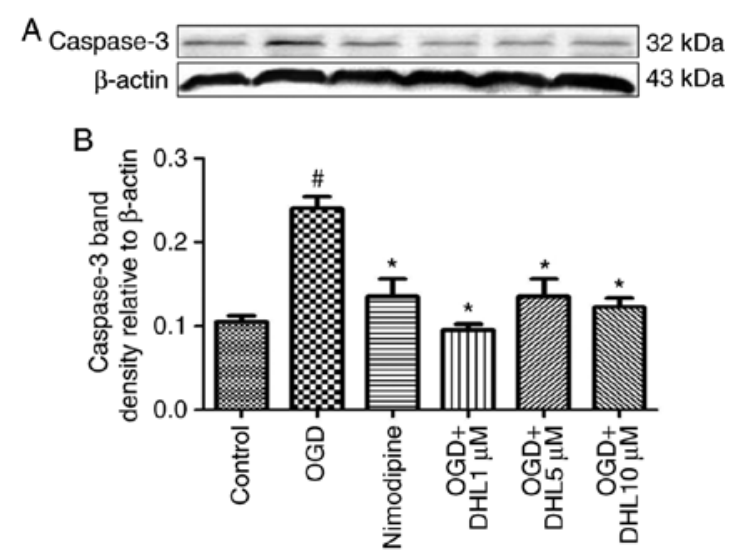

Figure 8. Effects of DHL on the expression of caspase-3. (A) Western blot analysis of caspase-3, with $\beta$-actin is shown as a loading control. (B) Quantitative analysis of the expression of caspase-3. Values are presented as the mean \pm standard deviation $(n=10) .{ }^{\#} \mathrm{P}<0.05$, compared with the control group; ${ }^{*} \mathrm{P}<0.05$, compared with the OGD/R group. DHL, dehydrocostuslactone; OGD/R, oxygen-glucose deprivation/reoxygenation.

decreased, and the expression levels of Bax and caspase-3 are increased in rats with cerebral ischemia injury $(39,40)$. Cysteine-requiring aspartate proteases are a family of proteases that are involved in apoptosis in vivo and in vitro $(41,42)$. Based on its functionality, the upstream pro-apoptotic factor caspase-9 becomes activated when a cell suffers from injury; the downstream factor caspase-7 and apoptosis of the executive factor caspase- 3 are further stimulated, and various cells consequently undergo apoptosis $(43,44)$. Secondary messengers, including $\mathrm{Ca}^{2+}, \mathrm{Bcl}-2$ and $\mathrm{Bax}$, are also activated when an apoptotic signal is delivered to the mitochondria (45). As a result, cyt-c is released from the mitochondria, and apaf-1 and caspase-9 combine to form a complex (46). This activates caspase-9 and other downstream effector factors, including caspases-3, caspase-6 and caspase-7; cell apoptosis is induced as a result (45). Consistent with previous reports, the present study demonstrated that the protein expression levels of Bcl-2 
A
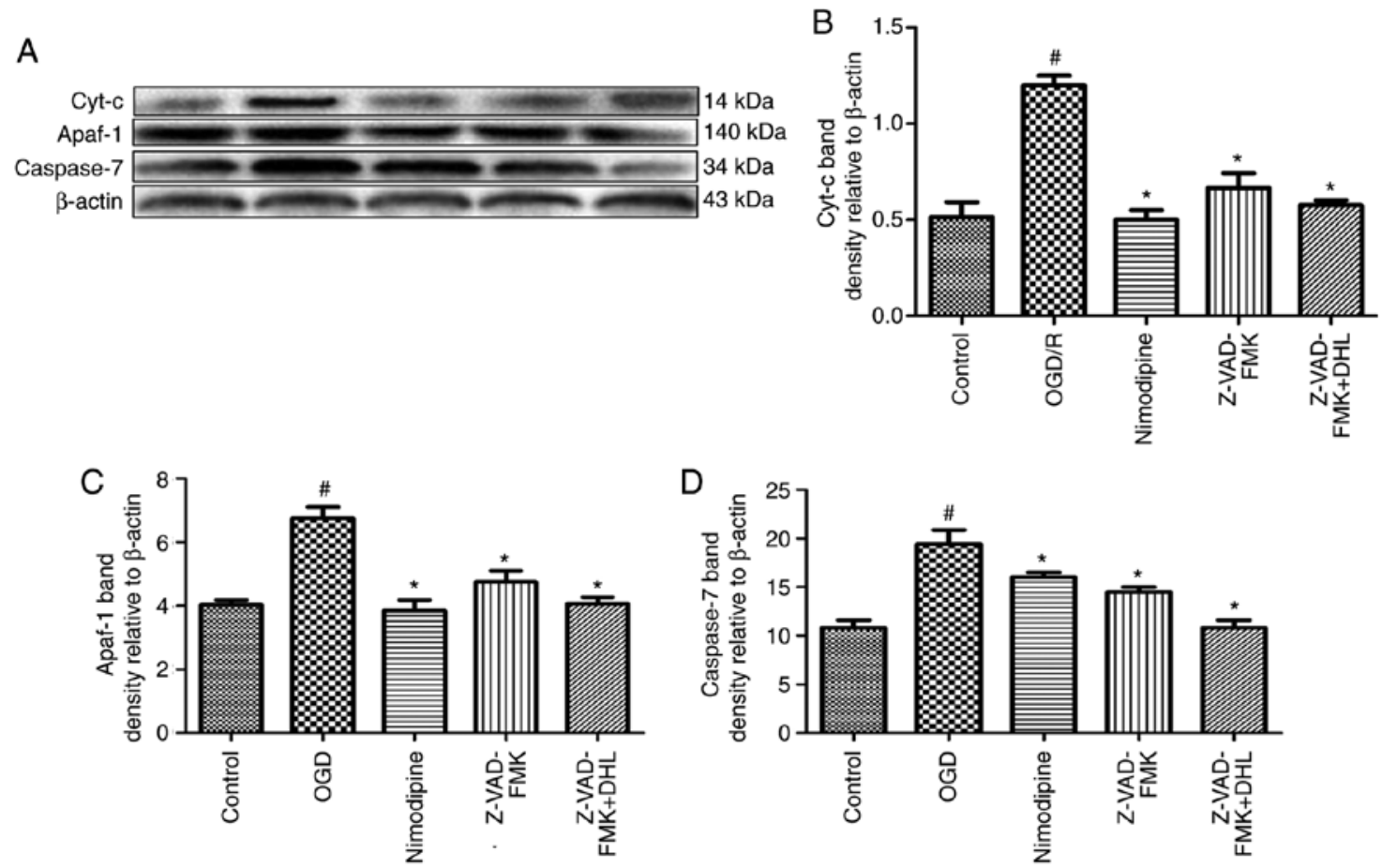

Figure 9. Effects of Z-VAD-FMK and DHL on the expression of cyt-c, apaf-1 and caspase-7. (A) Western blot analysis of f cyt-c, apaf-1 and caspase-7, with $\beta$-actin shown as a loading control. Quantitative analysis of the expression of (B) cyt-c, (C) apaf-1 and (D) caspase-7. Values are presented as the mean \pm standard deviation $(\mathrm{n}=10)$. ${ }^{\#} \mathrm{P}<0.05$, compared with the control group; ${ }^{*} \mathrm{P}<0.05$, compared with the OGD/R group. cyt-c, cytochrome $c$; apaf-1, apoptotic protease activating factor 1 ; DHL, dehydrocostuslactone; OGD/R, oxygen-glucose deprivation/reoxygenation.
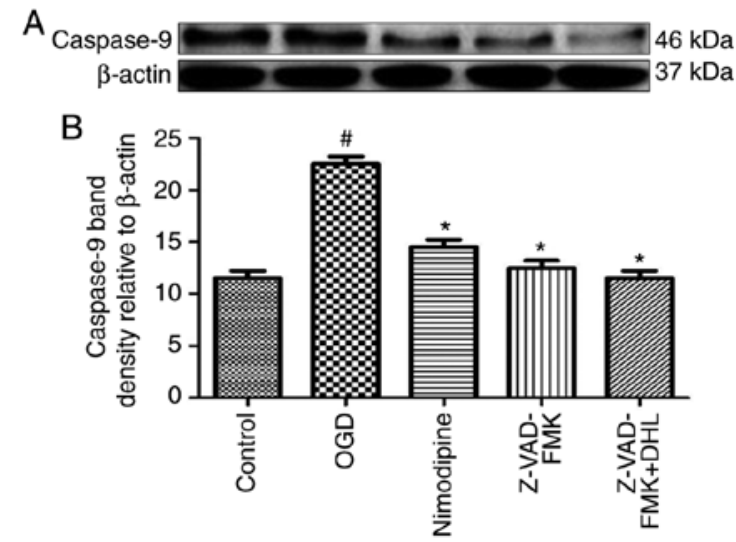

Figure 10. Effects of Z-VAD-FMK and DHL on the expression of caspase-9. (A) Western blot analysis of caspase-9, with $\beta$-actin shown as a loading control. (B) Quantitative analysis of the expression of caspase-9. Values are presented as the mean \pm standard deviation $(\mathrm{n}=10)$. ${ }^{\text {" }} \mathrm{P}<0.05$, compared with the control group; ${ }^{*} \mathrm{P}<0.05$, compared with the OGD/R group. DHL, dehydrocostuslactone; OGD/R, oxygen-glucose deprivation/reoxygenation.

were markedly decreased, and the protein expression levels of Bax, cyt-c, apaf-1, caspase-9, caspase-7 and caspase- 3 were increased when the cells were exposed to OGD/R. Treatment with DHL upregulated the expression levels of Bcl-2, and downregulated the expression levels of Bax, cyt-c, apaf-1, caspase-9, caspase-7 and caspase-3. Z-VAD-FMK, a widely irreversible caspase inhibitor, was used in the present study, and the results suggested that Z-VAD-FMK downregulated the expression levels of caspase-9, caspase-7 and caspase-3. DHL treatment also downregulated caspase- 9 , caspase-7 and caspase-3 to a greater extent than Z-VAD-FMK treatment.
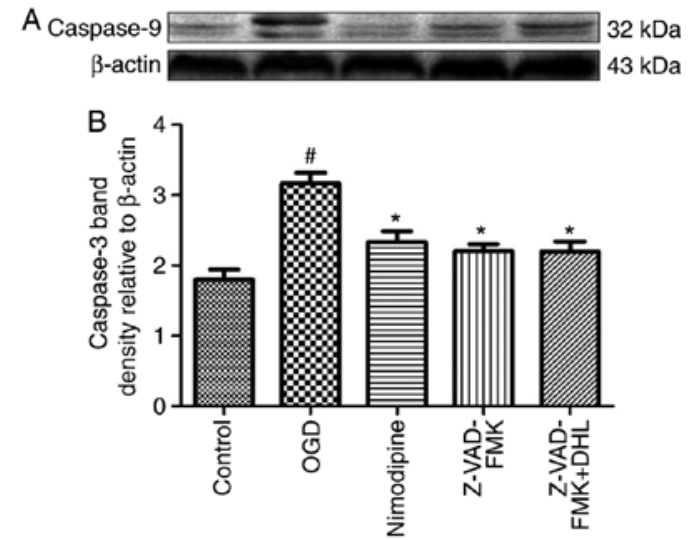

Figure 11. Effects of Z-VAD-FMK and DHL on the expression of caspase-3. (A) Western blot analysis of caspase-3, with $\beta$-actin shown as a loading control. (B) Quantitative analysis of the expression of caspase-3. Values are presented as the mean \pm standard deviation $(\mathrm{n}=10)$. ${ }^{\#} \mathrm{P}<0.05$, compared with the control group; ${ }^{*} \mathrm{P}<0.05$, compared with the OGD/R group. DHL, dehydrocostuslactone; $\mathrm{OGD} / \mathrm{R}$, oxygen-glucose deprivation/reoxygenation.

Autophagy is a programmed and physiologically conserved self-degradation process involved in focal cerebral infarction $(47,48)$. However, whether autophagy promotes neuronal death in response to OGD/R remains to be elucidated. In the present study, LC3 and SQSTM1, which are specific markers of autophagosomes, were probed to determine whether autophagic activity is induced by DHL treatment. LC3 is used as an autophagosome marker. Upon the induction of autophagy, LC3-I becomes converted to LC3-II, which then translocates to autophagosome membranes. The ratio of LC3-II to LC3-I is increased in mice subjected to middle cerebral artery 

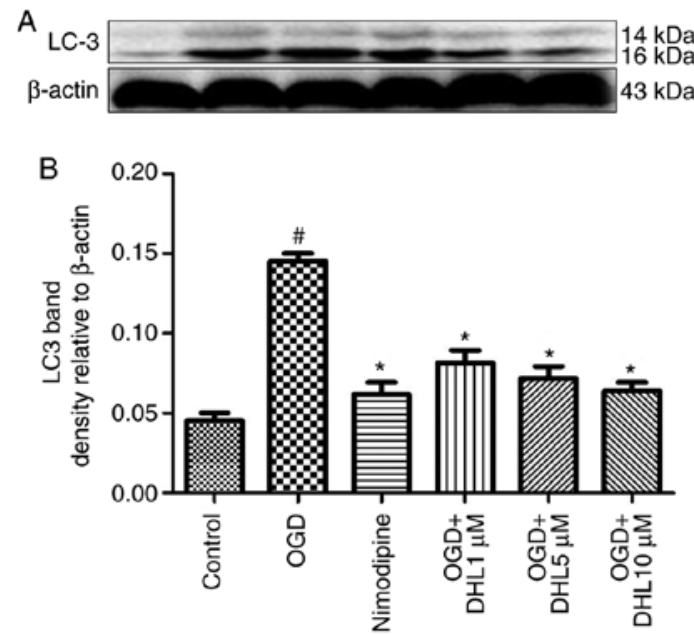

Figure 12. Effects of DHL on the expression of LC3-II. (A) Western blot analysis of LC3- II, with $\beta$-actin shown as a loading control. (B) Quantitative analysis of the expression of LC3-II. Values are presented as the mean \pm standard deviation $(\mathrm{n}=10) .{ }^{*} \mathrm{P}<0.05$, compared with the control group; ${ }^{*} \mathrm{P}<0.05$, compared with the OGD/R group. LC3, microtubule-associated protein 1 light chain 3; DHL, dehydrocostuslactone; OGD/R, oxygen-glucose deprivation/reoxygenation.
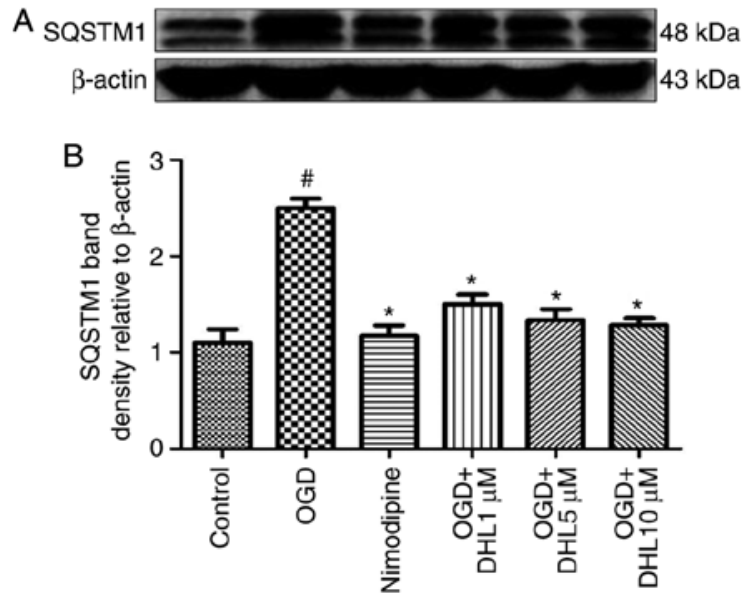

Figure 13. Effects of DHL on the expression of SQSTM1. (A) Western blot analysis of SQSTM1, with $\beta$-actin shown as a loading control. (B) Quantitative analysis of the expression of SQSTM1. Values are presented as the mean \pm standard deviation $(\mathrm{n}=10) .{ }^{*} \mathrm{P}<0.05$, compared with the control group; "P $<0.05$, compared with the OGD/R group. SQSTM1, sequestosome 1; DHL, dehydrocostuslactone; OGD/R, oxygen-glucose deprivation/reoxygenation.

occlusion (49). SQSTM1 (p62) is a ubiquitin-binding protein involved in autophagy. SQSTM1 binds to the autophagosomal membrane protein LC3 and carries SQSTM1-containing protein aggregates to autophagosomes. The level of SQSTM1 is used to monitor autophagic flux $(46,50)$. In the present study, LC3-II and SQSTM1 were markedly increased in the rat hippocampal slices injured by OGD/R. This result suggested that autophagy was activated in the rat hippocampal slices exposed to OGD/R, and this change was reversed by DHL treatment.

In the present study, the desired results were not obtained in paraffinized sections, frozen sections or flow cytometry. The results in the paraffinized sections were unsatisfactory due to the smaller and thinner hippocampal sample size $(400 \mu \mathrm{m})$, and there were more ice crystals in the frozen sections. Based on these reasons, it was not possible not evaluate the region or degree of hippocampal slice injury with hematoxylin and eosin staining, immunohistochemistry or TUNEL detection. Finally, the present study aimed to evaluate the degree of damage in the hippocampal slices using flow cytometry; the Annexin V/PI staining showed that the majority of cells were dead, and this cell death may have been induced by the preparation process of producing a single-cell suspension from the hippocampal slice.

Despite the limitations of the present study, the results revealed that DHL provided protective effects against hippocampal slice injury induced by OGD/R through anti-apoptotic and anti-autophagic effects. However, further investigations are required to determine other underlying mechanisms.

\section{Acknowledgements}

Not applicable.

\section{Funding}

The authors would like to acknowledge the financial support provided by the National Natural Science Foundation of Ningxia (grant no. NZ16078), the Ningxia College First-Class Discipline Construction Project (Chinese Medicine) Funded Project (grant no. NXYLXK2017A06) and the National Natural Science Foundation of China (grant nos. 81660700 and 81260679).

\section{Availability of data and materials}

The analyzed data sets generated during the study are available from the corresponding author upon reasonable request.

\section{Authors' contributions}

QZ, AC and XW established the hippocampal slice injury model and performed western blotting analysis. YuZ and YH analyzed and interpreted the data. ZZ performed lactate dehdrogenase activity measurement and tetrazolium chloride staining, and wrote the manuscript. YaZ and SR designed the study, supervised the research group and revised the manuscript critically for important intellectual content. The final version of the manuscript has been read and approved by all authors.

\section{Ethics approval and consent to participate}

This study was approved by the Ethics Committee of Ningxia Medical University (Yinchuan, China).

\section{Consent for publication}

Not applicable.

\section{Competing interests}

The authors declare that they have no competing interests. 


\section{References}

1. Dávalos A, Toni D, Iweins F, Lesaffre E, Bastianello S and Castillo J: Neurological deterioration in acute ischemic stroke: Potential predictors and associated factors in the European cooperative acute stroke study. Stroke 30: 2631-2636, 1999.

2. Wang Q, Tang XN and Yenari MA: The inflammatory response in stroke. J Neuroimmunol 184: 53-68, 2007.

3. Vssuvanish K, Gopalakrishnan A, Nazıroğlu M and Rajanikant GK: Calcium ion-the key player in cerebral ischemia. Curr Med Chem 21: 2065-2075, 2014.

4. Doyle KP, Simon RP and Stenzel-Poore MP: Mechanisms of ischemic brain damage. Neuropharmacology 55: 310-318, 2008.

5. Barone FC and Feuerstein GZ: Inflammatory mediators and stroke: New opportunities for novel therapeutics. J Cereb Blood Flow Metab 19: 819-834, 1999.

6. Li D, Liu M, Tao TQ, Song DD, Liu XH and Shi DZ: Panax quinquefolium saponin attenuates cardiomyocyte apoptosis and opening of the mitochondrial permeability transition pore in a rat model of ischemia/reperfusion. Cell Physiol Biochem 34 1413-1426, 2014

7. Simon HU, Haj-Yehia A and Levi-Schaffer F: Role of reactive oxygen species (ROS) in apoptosis induction. Apoptosis 5: 415-418, 2000.

8. Hengardner MO: Insight review articles: The biochemistry of apoptosis. Nature 207: 770-776, 2000.

9. Wu KJ, Huang JM, Zhong HJ, Dong ZZ, Vellaisamy K, Lu JJ, Chen XP, Chiu P, Kwong DWJ, Han QB, et al: A natural product-like JAK2/STAT3 inhibitor induces apoptosis of malignant melanoma cells. PLoS One 12: e0177123, 2017.

10. Leung CH, He HZ, Liu LJ, Wang MD, Chan DSH and Ma DL: Metal complexes as inhibitors of transcription factor activity. Coord Chem Rev 257: 3139-3151, 2013.

11. Wu KJ, Zhong HJ, Li G, Liu C, Wang HD, Ma DL and Leung CH: Structure-based identification of a NEDD8-activating enzyme inhibitor via drug repurposing. Eur J Med Chem 143: 1021-1027, 2017.

12. Kirino T, Tamura A and Sano K: Selective vulnerability of the hippocampus to ischemia-reversible and irreversible types of ischemic cell damage. Prog Brain Res 63: 39-58, 1985.

13. Obeidat AS, Jarvis CR and Andrew RD: Glutamate does not mediate acute neuronal damage after spreading depression induced by $\mathrm{O}_{2} /$ glucose deprivation in the hippocampal slice. J Cereb Blood Flow Metab 20: 412-422, 2000.

14. Zhan RZ1, Qi S, Wu C, Fujihara H, Taga K and Shimoji K: Intravenous anesthetics differentially reduce neurotransmission damage caused by oxygen-glucose deprivation in rat hippocampal slices in correlation with N-methyl-D-aspartate receptor inhibition. Crit Care Med 29: 808-813, 2001.

15. Sick TJ, Solow EL and Roberts EL: Extracellular potassium ion activity and electrophysiology in the hippocampal slice: Paradoxical recovery of synaptic transmission during anoxia. Brain Res 418: 227-234, 1987.

16. Schurr A, Payne RS, Heine M and Rigor BM: Hypoxia, excitotoxicity, and neuroprotection in the hippocampal slice preparation. J Neurosci Methods 59: 129-138, 1995.

17. Schurr A, Payne RS and Rigor BM: Protection by MK- 801 against hypoxia-, excitotoxin-, and depolarization-induced neuronal damage in vitro. Neurochem Int 26: 519-525, 1995.

18. Krnjević K: Electrophysiology of cerebral ischemia. Neuropharmacology 55: 319-333, 2008.

19. Zheng H, Chen Y, Zhang J, Wang L, Jin Z, Huang H, Man S and Gao W: Evaluation of protective effects of costunolide and dehydrocostuslactone on ethanol-induced gastric ulcer in mice based on multi-pathway regulation. Chem Biol Interact 250: 68-77, 2016.

20. Park EJ, Park SW, Kim HJ, Kwak JH, Lee DU and Chang KC: Dehydrocostuslactone inhibits LPS-induced inflammation by p38MAPK-dependent induction of hemeoxygenase- 1 in vitro and improves survival of mice in CLP-induced sepsis in vivo. Int. Immunopharmacol 22: 332-340, 2014.

21. Lee HK, Song HE, Lee HB, Kim CS, Koketsu M, Ngan LT and Ahn YJ: Growth inhibitory, bactericidal, and morphostructural effects of dehydrocostus lactone from Magnolia sieboldii Leaves on antibiotic-susceptible and -resistant strains of Helicobacter pylori. PLoS One 9: e95530, 2014.

22. Lin X, Peng Z and Su C: Potential anti-cancer activities and mechanisms of costunolide and dehydrocostuslactone. Int. J Mol Sci 16: 10888-10906, 2015
23. Park HJ, Kwon SH, Han YN, Choi JW, Miyamoto K, Lee SH and Lee KT: Apoptosis-Inducing costunolide and a novel acyclic monoterpene from the stem bark of Magnolia sieboldii. Arch Pharm Res 24: 342-348, 2001.

24. Hung JY, Hsu YL, Ni WC, Tsai YM, Yang CJ, Kuo PL and Huang MS: Oxidative and endoplasmic reticulum stress signaling are involved in dehydrocostuslactone-mediated apoptosis in human non-small cell lung cancer cells. Lung Cancer 68: 355-365, 2010

25. Zhang H, Schools GP, Lei T, Wang W, Kimelberg HK and Zhou M: Resveratrol attenuates early pyramidal neuron excitability impairment and death in acute rat hippocampal slices caused by oxygen-glucose deprivation. Exp Neurol 212: 44-52, 2008.

26. Huang XJ, Li Q, Zhang YP, Lü Q, Guo LJ, Huang L and He Z: Neuroprotective effects of cactus polysaccharide on oxygen and glucose deprivation induced damage in rat brain slices. Cell Mol Neurobiol 28: 559-568, 2008

27. Huang XJ, Li Q, Zhang YP, Lü Q, Guo LJ, Huang L and He Z: Neuroprotective effects of cactus polysaccharide on oxygen and glucose deprivation induced damage in rat brain slices. Brain Res Bull 87: 521-525, 2012.

28. Tagliari B, Zamin LL, Salbego CG, Netto CA and Wyse AT: Hyperhomocysteinemia increases damage on brain slices exposed to in vitro model of oxygen and glucose deprivation: Prevention by folic acid. Dev Neurosci 24: 285-291, 2006.

29. Cechetti F, Rhod A, Simão F, Santin K, Salbego C, Netto CA and Siqueira IR: Effect of treadmill exercise on cell damage in rat hippocampal slices submitted to oxygen and glucose deprivation. Brain Res 1157: 121-125, 2007.

30. Renic M, Kumar SN, Gebremedhin D, Florence MA, Gerges NZ, Falck JR, Harder DR and Roman RJ: Protective effect of 20-HETE inhibition in a model of oxygen-glucose deprivation in hippocampal slice cultures. Am J Physiol Heart Circ Physiol 302: H1285-H1293, 2012.

31. Preston E and Webster J: Spectrophotometric measurement of experimental brain injury. J Neurosci Methods 94: 187-192, 2000.

32. Mathews KS, McLaughlin DP, Ziabari LH, Toner CC, Street PC, Hisgrove E, Bezzina EL and Stamford JA: Rapid quantification of ischemic injury and cerebroprotection in brain slices using densitometric assessment of 2,3,5-triphenyltetrazolium chloride staining. J Neurosci Methods 102: 43-51, 2000

33. Wang ZJ, Li GM, Nie BM, Lu Y and Yin M: Neuroprotective effect of the stearic acid against oxidative stress via phosphatidylinositol 3-kinase pathway. Chem Biol Interact 160: 880-887, 2006.

34. Zhao Q, Cheng X, Wang X, Wang J, Zhu Y and Ma X: Neuroprotective effect and mechanism of Mu-Xiang-You-Fang on cerebral ischemia-reperfusion injury in rats. J Ethnopharmacol 192: 140-147, 2016.

35. Lalonde CC and Mielke JG: Selective vulnerability of hippocampal sub-fields to oxygen-glucose deprivation is a function of animal age. Brain Res 1543: 271-279, 2014.

36. Adams JM and Cory S: The Bcl-2 protein family: Arbiters of cell survival. Science 281: 1322-1326, 1998.

37. Kitagawa K, Matsumoto M, Tsujimoto Y, Ohtsuki T, Kuwabara K, Matsushita K, Yang G, Tanabe H, Martinou JC, Hori M and Yanagihara T: Amelioration of hippocampal neuronal damage after global ischemia by neuronal overexpression of BCL-2 in transgenic mice. Stroke 29: 2616-2621, 1998

38. Misao J, Hayakawa Y, Ohno M, Kato S, Fujiwara T and Fujiwara H: Expression of bcl-2 protein, an inhibitor of apoptosis, and Bax, an accelerator of apoptosis, in ventricular myocytes of human hearts with myocardial infarction. Circulation 94: 1506-1512, 1996

39. van Delft MF and Huang DC: How the Bcl-2 family of proteins interact to regulate apoptosis. Cell Res 16: 203-213, 2006

40. Wang GH, Lan R, Zhen XD, Zhang W, Xiang J and Cai DF: An-Gong-Niu-Huang Wan protects against cerebral ischemia induced apoptosis in rats: Up-regulation of Bcl-2 and down-regulation of Bax and caspase-3. J Ethnopharmacol 154: 156-162, 2014.

41. Cao G, Minami M, Pei W, Yan C, Chen D, O'Horo C, Graham SH and Chen J: Intracellular Bax translocation after transient cerebral ischemia: Implications for a role of the mitochondrial apoptotic signaling pathway in ischemic neuronal death. J Cereb Blood Flow Metab 21: 321-333, 2001.

42. Riedl SJ and Shi Y: Molecular mechanisms of caspase regulation during apoptosis. Nat Rev Mol Cell Biol 5: 897-907, 2004. 
43. García de la Cadena S and Massieu L: Caspases and their role in inflammation and ischemic neuronal death. Focus on caspase-12. Apoptosis 21: 763-777, 2016.

44. Zhu L, Yuan H, Guo C, Lu Y, Deng S, Yang Y, Wei Q, Wen L and He Z: Zearalenone induces apoptosis and necrosis in porcine granulosa cells via a caspase-3- and caspase-9-dependent mitochondrial signaling pathway. J Cell Physiol 227: 1814-1820, 2012.

45. Bhuiyan MS and Fukunaga K: Inhibition of HtrA2/Omi ameliorates heart dysfunction following ischemia/reperfusion injury in rat heart in vivo. Eur J. Pharmacol 557: 168-177, 2007.

46. Li Y, Zhang J, Chen L, Xing S, Li J, Zhang Y, Li C, Pei Z and Zeng J: Ebselen reduces autophagic activation and cell death in the ipsilateral thalamus following focal cerebral infarction. Neurosci Lett 600: 206-212, 2015.

47. Solá S, Morgado AL and Rodrigues CM: Death receptors and mitochondria: Two prime triggers of neural apoptosis and differentiation. Biochim Biophys Acta 1830: 2160-2166, 2013.
48. Xing S, Zhang Y, Li J, Zhang J, Li Y, Dang C, Li C, Fan Y, Yu J, Pei $Z$ and Zeng JS: Beclin 1 knockdown inhibits autophagic activation and prevents the secondary neurodegenerative damage in the ipsilateral thalamus following focal cerebral infarction. Autophagy 8: 63-76, 2012.

49. Zhang J, Zhang Y, Li J, Xing S, Li C, Li Y, Dang C, Fan Y, $\mathrm{Yu}$ J, Pei Z and Zeng JS: Autophagosomes accumulation is associated with $\beta$-amyloid deposits and secondary damage in the thalamus after focal cortical infarction in hypertensive rats. J Neurochem 120: 564-573, 2012.

50. Jiang P and Mizushima N: LC3- and p62-based biochemical methods for the analysis of autophagy progression in mammalian cells. Methods 75: 13-18, 2015. 